\author{
Giuseppe Nappi \\ Alfredo Costa \\ Cherubino Di Lorenzo \\ Alberto Proietti Cecchini \\ Ennio Pucci \\ Giorgio Sandrini
}

\section{Chronic daily headache: old problems, new vistas}

G. Nappi (西) • A. Costa • C. Di Lorenzo • A. Proietti Cecchini • E. Pucci • G. Sandrini University Centre for Adaptive Disorders and Headache,

C. Mondino Foundation,

Via Palestro 3, I-27100 Pavia, Italy

e-mail: nappi@mondino.it

Tel.: +39-0382-380202

Fax: +39-0382-380311

\section{G. Nappi}

Institute of Nervous and Mental Diseases, University of Rome La Sapienza, Rome, Italy

\begin{abstract}
In 1988 the problems concerning chronic daily headache $(\mathrm{CDH})$ were neglected by the classification of the International Headache Society (IHS). More than ten years later, this issue is still debated, also in light of the foreseen revised classification. Several terms have been used to define the clinical picture of $\mathrm{CDH}$, and different criteria have been proposed for the diagnosis of these forms. In most cases, CDH appears to evolve from an episodic migraine, but the temporal limits between an episodic and a no-longer episodic form of migraine are questionable. A decreased threshold for
\end{abstract}

headache recurrence in $\mathrm{CDH}$ is currently hypothesized, and it may be due to either an impaired control system or a sensitization of the trigeminal neurons, occurring regardless of the original nature of headache. The identification of genetic alterations and neurobiological changes underlying the different forms of $\mathrm{CDH}$ may greatly facilitate any nosographic and therapeutical approach to this broad spectrum of disorders.

Key words Chronic daily headache . Tension-type headache $\cdot$ Migraine • Drug abuse $\cdot$ Nosography

\section{Introduction}

The 1988 classification of the International Headache Society (IHS) represented a considerable advance in the nosography of primary headaches. However, the issue of chronic daily headache (CDH) was not fully addressed, and as a revised version of the classification is just around the corner, the need to settle this controversial matter is pressing. $\mathrm{CDH}$ is in fact a descriptive term for a heterogeneous and poorly defined clinical group of conditions characterized by headache present more than half the time, often on a daily (or nearly daily) basis.

Even though it is a rare condition, affecting $0.5 \%-5 \%$ of the population according to different reports [1-3], $\mathrm{CDH}$ accounts for the majority of patients seeking treatment in headache centers [4]. This clinical form of headache is often a major therapeutic challenge for the physician who, in this case more than in others, is faced with a complex disorder whose treatment and outcome may be successfully improved only through a multilevel approach. Indeed, both pharmacological and non-pharmacological strategies are available to tackle both environmental factors and the individual's abnormal coping with stressors. In addition, psychiatric and cerebrovascular comorbidity, as well as the development of mood and anxiety disturbances secondary to increased migraine frequency and drug abuse should be borne in mind in the clinical and therapeutical management of $\mathrm{CDH}$.

\section{Nosographic aspects of CDH}

Difficulties over terminology have always clouded the picture of $\mathrm{CDH}$. Indeed, definitions like "evolutive migraine" 
and "migraine with interparoxysmal headache" $(\mathrm{MIH})$ are still being used to refer to otherwise ill-described forms of continuous or subcontinuous headache originally of a migrainous nature. The panoply of these terms is illustrated in Fig. 1.

From the phenotypical perspective, as the frequency of migraine attacks increases a blunting of the clinical features may be observed (in particular, a decrease in pain intensity) until a condition is ultimately produced in which the single features are lost or mixed, and where criteria for both chronic tension-type headache and migraine may be fulfilled. This is why, in efforts to obtain a correct nosographic definition of $\mathrm{CDH}$, a purely descriptive approach appears to be inadequate.

Several recent studies have demonstrated that $\mathrm{CDH}$ patients diagnosed according to the current IHS criteria suffer from chronic tension-type headache (CTTH) with migraine being a possible additional diagnosis. However, if one takes into account the natural history and the evolution of the disorder, it emerges that most patients in fact do not have two but one single condition, i.e. a migraine form whose features have changed over the years. In these cases, the frequency of the attacks has increased to the point where a chronic headache has replaced the previous episodic form, with or without the occurrence of full blown migraine attacks.

This temporal profile is different from cases where sin- gle acute headache attacks are initially of the tension type, later becoming throbbing in quality and evolving into a clear migraine, referred to as "mixed headache". Re-examination of the old dispute over whether migraine and tension-type headache are two distinct entities [5] or a single one whose clinical signs and symptoms appear along a spectrum or continuum of varying intensity [6] is probably beside the point. Indeed, even though the two forms derive from a different "tree" (as genetics now suggests), the involvement of the trigeminal nucleus and the central antinociceptive system is likely to represent a final route, common two both disorders, responsible for the clinical expression of pain. The occurrence of a sensitization of trigeminal neurons or an impairment in pain control system due to the recurrence of either migraine or TTH attacks may constitute the common neurobiological substrate of $\mathrm{CDH}$.

The studies by Mathew [7], Sandrini et al. [8] and Manzoni et al. [9] are all consistent in reporting that more than two-thirds of $\mathrm{CDH}$ patients reaching headache centers suffer from a form of headache that has evolved from migraine. By contrast, in a recent population-based study, it emerged that only one-third of $\mathrm{CDH}$ patients were migraineurs in origin [3]. However, this discrepancy may be explained by a bias in the first three studies [7-9] with regard to the selection of the patients, who had been enrolled in specialized headache clinics.

Fig. 1 The terms in the "can" are the definitions, some of which are still in use, for $\mathrm{CDH}$ when this represents a nonepisodic or no longer episodic form of migraine

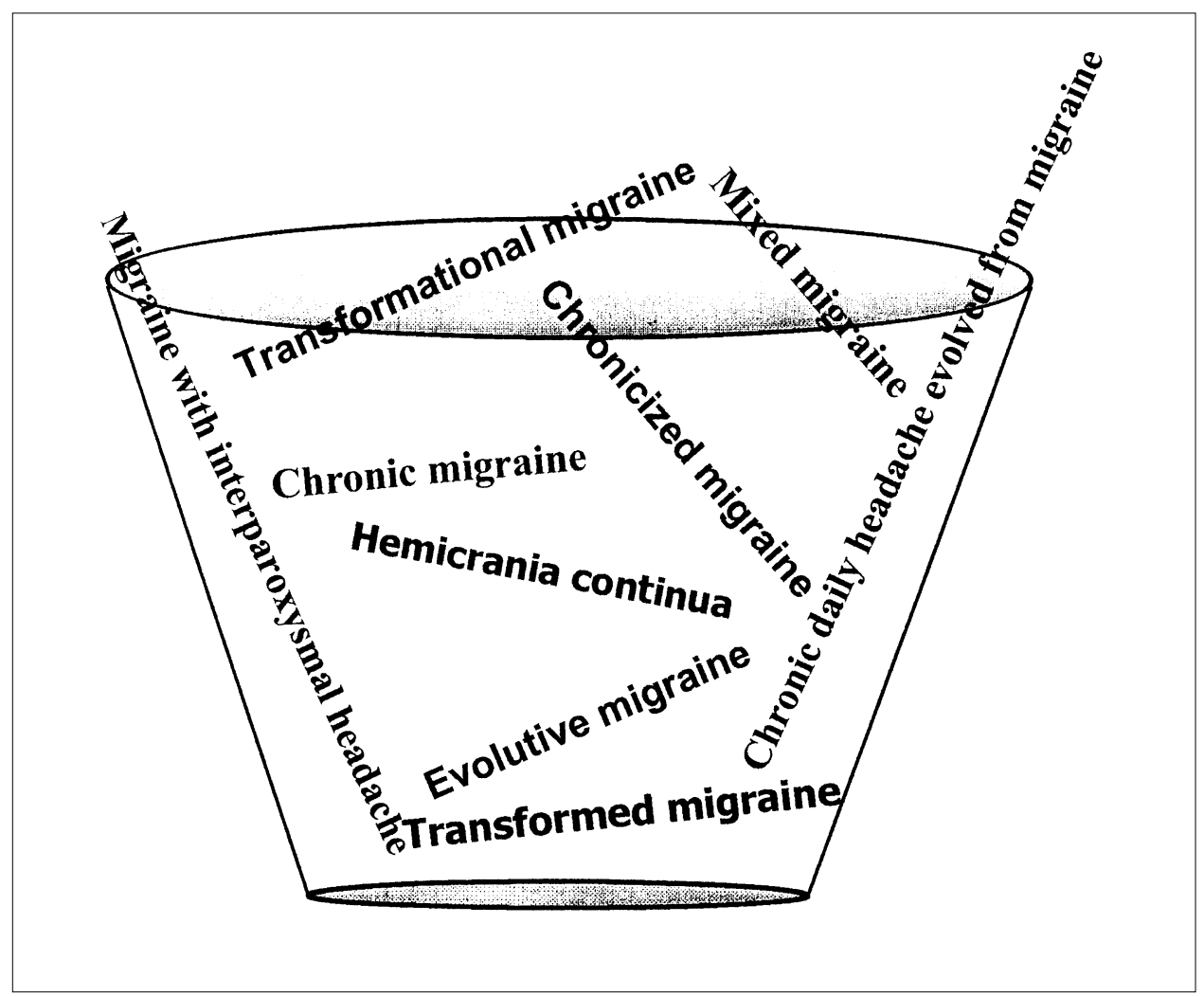


On the other hand, it is now clear that at least one-third of $\mathrm{CDH}$ cases cannot be diagnosed using the current IHS classification [9, 10]. The existence of this subgroup of unclassifiable headache patients appears to be due to either the presence of associated migrainous features which do not fulfil the criteria for CTTH or to a continuous headache, incompatible with the diagnosis of migraine.

The development of $\mathrm{CDH}$ from a pre-existing form of migraine deserves further consideration. Migraine is a chronic illness interspersed with acute signs and symptoms, and although it is currently defined in terms of attacks according to IHS criteria, it displays a considerable variability in the way it manifests itself in the single individual and especially in different individuals. Whether migraine is considered from the point of view of the attacks (e.g. presence or absence of aura, degree of pain severity) or as a disease (e.g. pattern of onset, recurrence, association with other diseases, evolution, outcome), this heterogeneity is a feature that consistently underpins it. For these reasons, migraine can be currently viewed as a "complex disease" [11]. In most cases, migraine has a multifactorial, inherited character: such level of complexity, however, is further increased by the effect of modifying genes (i.e. those encoding dopamine receptors), by comorbidity, and by the fact that the expression of comorbidity varies over time. The importance of this phenomenon, which may be referred to as "phenotypical heterochronia", emerges upon simple observation of the natural history of migraine in the lifetime of different individuals. The phenotypical manifestations remain unchanged over the years in some patients, while in others the clinical picture becomes more complicated, and may include arterial hypertension (per se a risk factor for cerebrovascular accidents), anxiety and mood disturbances. On the other hand, it is well known that the presence of hypertension and psychiatric disorders often facilitates changes in the migraine pattern, resulting in forms of $\mathrm{CDH}[7,11]$.

The reciprocal links between migraine and its associated diseases remain obscure. Similarly, the factors affecting the variable evolution of the clinical picture deserve further

Table 1 Proposed headache classification and IHS codes for CDH (From [10] with permission)

$\mathrm{CDH}$, Daily or nearly daily headache lasting $>4 \mathrm{~h} /$ day for $>15$ days/month
1.8
2.2
Transformed migraine
4.7
Chronic tension-type headache
New daily persistent headache
4.8
Hemicrania continua

investigation, although it is likely that age and gender are among the most important ones $[12,13]$.

\section{CDH classification: new proposals}

The limits of the IHS classification with regard to $\mathrm{CDH}$ forms have been recognized, but it has to be said that several attempts have also been made to improve it. Solomon et al. [14] suggested calling the migraine subtype that evolves into $\mathrm{CDH}$ "transformational migraine", while Mathew [7] proposed that a further form, termed new daily persistent headache, be built into the new classification. In line with these proposals, Silberstein et al. provided tentative diagnostic criteria (Table 1) that included transformed migraine, chronic tension-type headache, new daily persistent headache and hemicrania continua, each of which may or may not be associated with pharmacological abuse [10]. Manzoni et al. [9] also suggested adding to the classification a new diagnostic category, "evolution of migraine", embracing two conditions: migraine with interparoxysmal headache (MIH) and chronic migraine (Table 2). The interparoxysmal headache, in turn, may or may not fulfil the criteria for CTTH.

With particular regard to the forms of migraine which are non-episodic or no longer episodic, one issue remains unresolved concerning the temporal limits, as shown in Figure 2. The term chronic migraine suggested by Manzoni et al. [9] implies the presence of headache for at least 6 days a week (24 days/month) for at least 1 year, while the definition transformed migraine proposed by Silberstein et al. [10] describes a daily or almost daily headache ( $>15$ days/month) present for at least 3 months. Thus, a question may be asked: where do we draw the line between a form of migraine which shows a high frequency of attacks, but is still episodic, and a chronic migraine? Indeed, in accordance with the current diagnostic criteria, there is general consensus that migraine attacks may last up to 72 hours.

Table 2 Proposed headache classification and IHS codes for $\mathrm{CDH}$ (From [9] with permission)

\begin{tabular}{|c|c|c|}
\hline \multicolumn{3}{|c|}{ Evolution of migraine } \\
\hline \multirow[t]{3}{*}{1.7 .1} & Migrain & with interparoxysmal headache \\
\hline & 1.7.1.1 & $\begin{array}{l}\text { Migraine with interparoxysmal } \\
\text { headache fulfilling the criteria for } \\
\text { chronic tension-type headache }\end{array}$ \\
\hline & 1.7.1.2 & $\begin{array}{l}\text { Migraine with interparoxysmal } \\
\text { headache not fulfilling the criteria for } \\
\text { chronic tension-type headache }\end{array}$ \\
\hline 1.7 .2 & Chronic & higraine \\
\hline
\end{tabular}


Fig. 2 The temporal criteria proposed for $\mathrm{CDH}$ need to be discussed further, as they may not help in distinguishing between a form of migraine with high frequency of attacks and a proper CDH. Migraine occurs with different attack frequency (left arrow). A high frequency, such as 5 attacks per month, each lasting for 3 days, would result in a total of 15 days with headache per month, thus overlapping with the proposed temporal limits for transformed migraine (right arrow). The suggested criteria for chronic migraine (at least 24 days with headache/month) appear to be more strict in this respect (middle arrow)

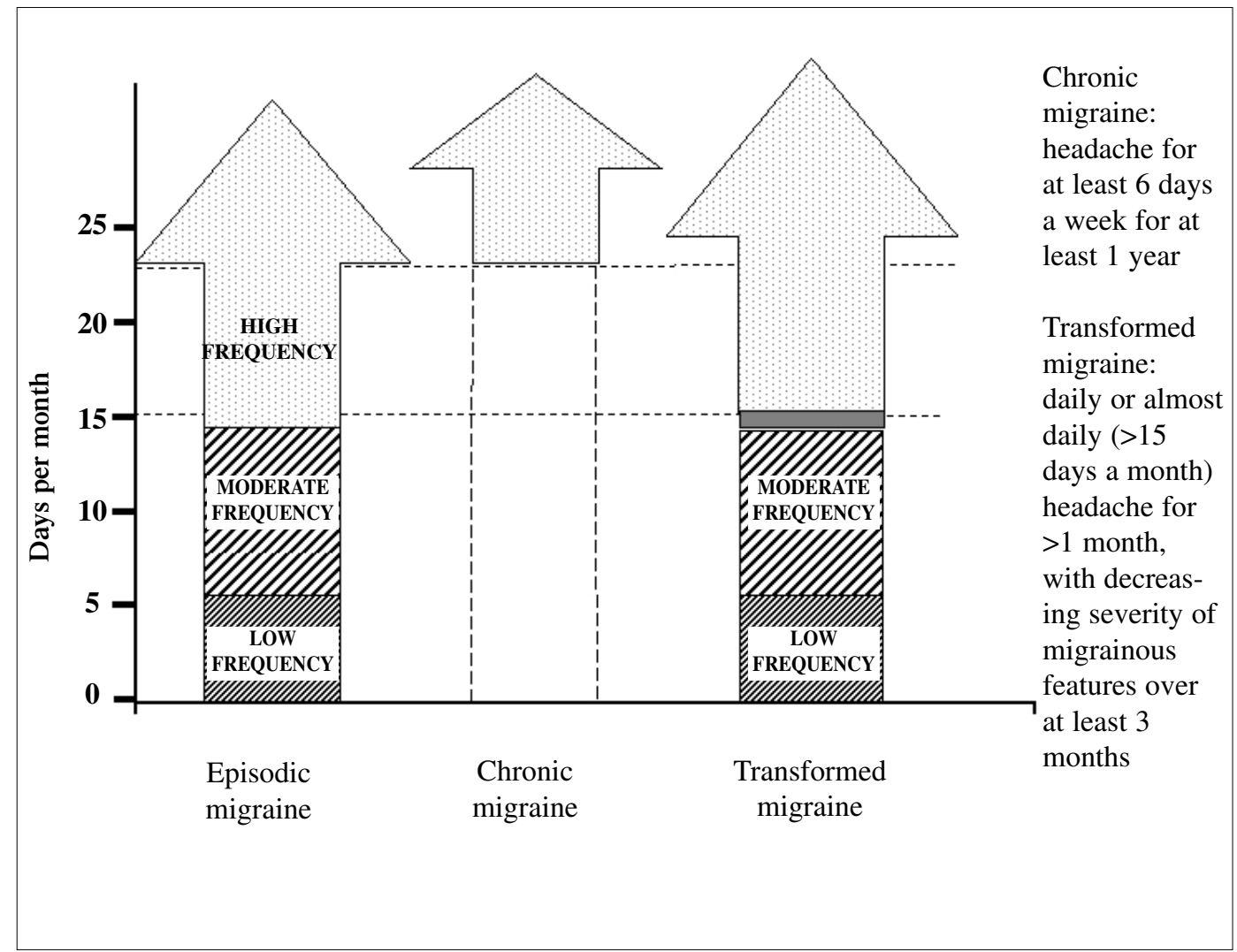

This means that a patient experiencing 8 attacks/month, none of which lasts less than 72 hours, would experience headache for a total of 24 days, the temporal limit for a "chronic migraine". Similarly, a patient reporting a frequency of 5 attacks/month, all lasting at least 72 hours, would consequently meet the criteria for a "transformed" (chronic) - rather than episodic - form of migraine. It has also to be borne in mind that headache centers commonly encounter patients with several attacks per month, and that $50 \%$ or more migraineurs experience pain attacks with moderate to high frequency [15].

These criteria therefore appear to be inadequate if we wish to obtain a precise definition of $\mathrm{CDH}$, even though, solely from the temporal point of view, better provision for the criteria proposed by Manzoni et al. [9] may be made in the future revised classification. Moreover, Goadsby has recently recommended greater care in the use of the term chronic migraine, maintaining that it should, as has so far been the case with other forms of chronic primary headache, be restricted to the transformed migraine forms [16].

In an epidemiological study on a general population, Rasmussen et al. [18] reported that the prevalence of migraineurs with additional TTH was as high as $83 \%$. This figure, however, was not significantly different from that found in non-migraineurs, supporting the view that migraine and TTH are two distinct disorders that, given the considerable prevalence of the latter one, can coexist by chance. However, the hypothesis of a simple association of migraine and CTTH no longer appears tenable. The coexistence of the two disorders is indeed much more common than might be expected on the basis of the epidemiological profile of each of them. This, again, points to the existence of a form of chronic migraine [16].

Furthermore, in migraine there appears to be no direct correlation between frequency and severity of attacks, whereas these two aspects are likely to be correlated in TTH. In this respect, a double-blind placebo-controlled study carried out by Lipton et al. [17] showed an equal efficacy of $50 \mathrm{mg}$ sumatriptan in both types of attacks in a group of patients with migraine alternating with disabling TTH, while the drug was found to be ineffective in patients suffering exclusively from TTH episodes.

All these considerations should be borne in mind when including $\mathrm{CDH}$ forms into the new IHS classification. In the meantime, $\mathrm{CDH}$ remains an enigma, but there is little doubt that the forms that come under this definition each present a peculiar natural history, and that only by obtaining precise information can a diagnosis be made [4, 19]. 


\section{Pathophysiological aspects of CDH}

It is clear that a chronic form of headache cannot be considered simply as a worsening, in terms of the frequency of the clinical manifestations, of the corresponding episodic form. There are, almost certainly, different pathophysiological mechanisms underlying acute and chronic pain. The chronic forms of headache, however, tend to share common pathophysiological processes, such as a derangement of pain control of central origin, with sensitization, to a variable degree, of second order trigeminal neurons and defective pain modulation. These aspects may account for the overlap in the clinical appearance of these disorders, and explain the phenomenon of gradual decrease in the threshold for headache recurrence [20]. The condition may be further complicated, in several regards, by the problem of drug misuse.

In recent functional neuroimaging studies [21, 22], primary neurovascular headaches (with clearly defined clinical features, such as cluster headache $(\mathrm{CH})$ and migraine), have been shown to display selective activation of brain regions specifically related to the clinical signs and symptoms (i.e. the midbrain in migraine and the hypothalamus in $\mathrm{CH}$ ). By contrast, the mild clinical profile of TTH may denote a sort of redundancy of central neurobiological processes which can result in a common clinical phenotype.

\section{CDH: different biotypes}

The role of genetic factors in migraine is well established. Recently, a familial component has also been demonstrated for CTTH [23]. The genetic predisposition to headache, first of all, affects the pattern of evolution and the natural history of the disorder, while an intense modulatory effect is provided by the complex interplay of environmental and psychosocial factors [11]. New instruments to define the various forms of $\mathrm{CDH}$ also from a neurobiological perspective are therefore needed. To this end, neurophysiological tests may help to differentiate between the various forms of $\mathrm{CDH}$. For instance, while a lack of habituation may be used as a marker of the migraine trait [24], the reduction of the exteroceptive suppression of the temporalis muscle (ES2) may biologically characterize TTH [25]. Without doubt, more direct insight into any predisposition to headache can be obtained from the genetic approach to patients. Different biotypes of $\mathrm{CDH}$, each requiring a different therapeutic approach, may thus be identified, as may comorbid conditions such as addictive behavior (painkiller misuse), and other psychopathological aspects [11].

\section{References}

1. Newman LC, Lipton RB, Solomon S, Stewart WF (1994) Daily headache in a population sample: results from the American Migraine Study. Headache 34(1):295

2. Scher AI, Stewart WF, Liberman J, Lipton RB (1998) Prevalence of frequent headache in a population sample. Headache 38:497-506

3. Castillo J, Munoz P, Guitera V, Pascual J (1999) Epidemiology of chronic daily headache in the general population. Headache 39:190-196

4. Silberstein SD, Lipton RB, Solomon S, Mathew NT (1994) Classification of daily and near daily headaches: proposed revisions to the HIS classification. Headache 34:1-7

5. Rasmussen BK (1996) Migraine and tension-type headache are separate disorders. Cephalalgia 16:217-223

6. Leston JA (1996) Migraine and tension-type headache are not separate disorders. Cephalalgia 16:220-222

7. Mathew NT (1993) Transformed migraine. Cephalalgia 1 3[Suppl 12]:78-83
8. Sandrini G, Manzoni GC, Zanferrari C, Nappi G (1993) An epidemiological approach to the nosography of chronic daily headache. Cephalalgia 13[Suppl 12]:72-77

9. Manzoni GC, Granella F, Sandrini G, Cavallini A, Zanferrari C, Nappi G (1995) Classification of chronic daily headache by International Headache Society criteria: limits and new proposals. Cephalalgia 15:37-43

10. Silberstein SD, Lipton RB, Sliwinski M (1996) Classification of daily and near-daily headaches: field trial of revised IHS criteria. Neurology 47:871-875

11. Nappi G, Costa A, Tassorelli C, Santorelli FM (2000) Migraine as a complex disease: heterogeneity, comorbidity and genotype-phenotype interactions. Funct Neurol 15:87-93

12. Carolei A, Marini C, De Matteis G (1996) History of migraine and risk of cerebral ischaemia in young adults. The Italian National Research Council Study Group on Stroke in the Young. Lancet 347:1503-1506
13. Breslau N, Kilcoat HD, Andreski P (1996) Further evidence on the link between migraine and neuroticism. Neurology 47:663-667

14. Solomon S, Lipton RB, Newman LC (1992) Evaluation of chronic daily headache-comparison to criteria for chronic tension-type headache. Cephalalgia 12:365-368

15. Stewart WF, Lipton RB, Celentano DD, Reed ML (1992) Prevalence of migraine headache in the United States. Relation to age, income, race, and other sociodemographic factors. JAMA 267(1):64-69

16. Goadsby P (1999) Chronic tensiontype headache: where are we? Brain 122:1611-1612

17. Lipton RB, Stewart WF, Cady R et al (2000) Sumatriptan for the range of headaches in migraine sufferers: results of the spectrum study. Headache 40:783-791

18. Rasmussen BK, Jensen R, Schroll M, Olesen J (1992) Interrelations between migraine and tension-type headache in the general population. Arch Neurol 49:914-918 
19. Nappi G, Granella F, Sandrini G, Manzoni GC (1999) Chronic daily headache. How should it be included in the IHS classification? Headache 39:197-203

20. Schoenen J, Olesen J (2000) Synthesis of tension-type headache mechansims. In: Olesen J, Tfelt Hansen P, Welch KMA (eds) The headaches, 2nd edn. Lippincott Williams \& Wilkins, Philadelphia, pp 615-618
21. Weiller C, May A, Limmroth V, Juptner M, Kaube H, Schayck RV, Coenen HH, Diener HC (1995) Brain stem activation in spontaneous migraine attacks. Nat Med 1(7):658-660

22. May A, Bahra A, Buchel C, Frackoviak RSJ, Goadsby PJ (1998) Hypothalamic activation in cluster headache attacks. Lancet 351:275-278

23. Russell BM, Ostergaard S, Bendsten L, Olesen J (1999) Familial occurrence of chronic tension-type headache. Cephalalgia 19:207-210
24. Schoenen J, Wang W, Albert A, Delwaide JP (1995) Potentiation instead of habituation characterizes visual evoked potentials in migraine patients between attacks. Eur J Neurol 2:115-122

25. Schoenen J, Jamart B, Gerard P, Lenarduzzi P, Delwaide JP (1987) Exteroceptive suppression of temporalis muscle activity in chronic headache. Neurology 37:1834-1836 